\title{
CT Prostate Segmentation Based on Continuously Updated Random Forests
}

\author{
Huangjian DENG ${ }^{1, a}$, Xiubin DAI ${ }^{1, b}$, Dandan $\mathrm{SHI}^{1, \mathrm{c}}$ \\ ${ }^{1}$ College of Geographic and Biologic Information, Nanjing University of Posts and \\ Telecommunications, Nanjing, Jiangsu, 210015 Chinas. \\ aemail: 13236528512@163.com, bemail: daixb@njupt.edu.cn, cemail:18252063295@163.com
}

Keywords: Prostate Segmentation; Treatment Images; Continuously Updated Random Forests

\begin{abstract}
It is important to segment prostate automatically in the daily treatment images. However, previous methods often ignore the previous segmented treatment images which contain valuable patient-specific information. To this end, this paper proposes a novel CT prostate segmentation method based on a random forest model which is trained as a classifier to segment prostates. This model can be continuously updated by adding newly segmented prostate shapes into the training pool. In this way, more patient-specific information is incorporated into the training procedure. The experimental results show that the proposed method can improve the accuracy of prostate segmentation efficiently.
\end{abstract}

\section{Introduction}

Image guided radiation therapy (IGRT) is an effective technology for prostate cancer radiation treatment and widely used in clinic [1]. Since clinicians are supposed to precisely deliver the radiation dose to the exact positions of prostate tumor(s) and minimize the dose to the neighboring healthy tissues at the same time, it is important to accurately and fast localize prostates in the daily treatment images.

However, low contrast and dramatic changing of appearance of treatment images across different treatment days make prostate segmentation in the treatment images difficult [2]. Besides, unpredicted daily prostate motions also increase the difficulty of prostate segmentation. Figure 1 displays quite different prostate appearance and shapes from different treatment days of the same patient.

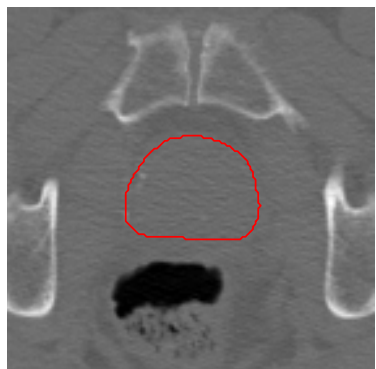

(a)

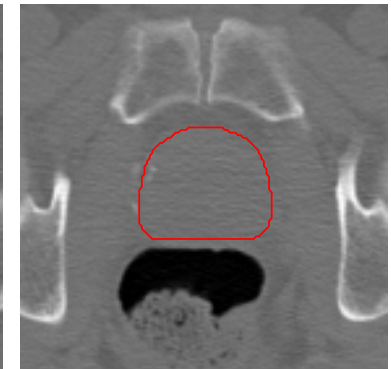

(b)

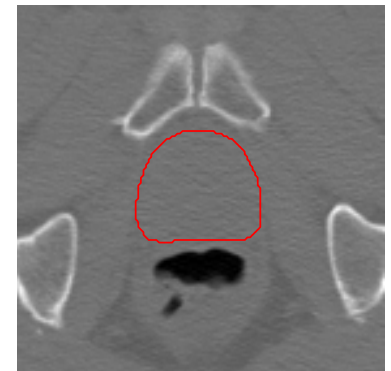

(c)

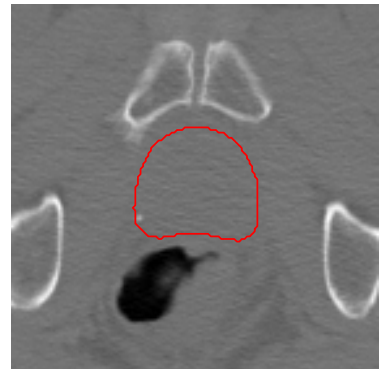

(d)

Fig.1. The same slices from different treatment days of the same patient. (a-d) show quite different prostate appearance and shapes. Here, the red contours denote the prostate shapes.

To overcome the aforementioned challenges, numerous researches have been conducted, in which deformable model based methods play an important role and have shown good performance. For example, Costa et al. [3] built coupled 3D deformable models by using the non-overlapping constraint between the prostate and bladder. Freeman [4] segmented prostates on the basis of the matched probability distributions of photometric variables. In Feng's work, the gradient profile features and region-based features were combined together to guide the deformable segmentation [5]. Nevertheless, these deformable model based methods are highly depended on appropriate initialization of the model, which makes their performance limited. 
Additionally, deformable registration based methods have also been extensively studied. Liao et al. [6] used patient-specific information to present a feature-guided deformable registration method. To increase accuracy, Foskey et al.[7] proposed a deflation method to eliminate bowel gas before registration. Since deformable registration based methods consume more time than deformable model based methods, they are not generally suitable for the clinical practice.

Recently, many researchers start to apply the machine learning method in the field of prostate segmentation. The main idea of these classification-based methods is to train a series of classifiers which will be used to identify if the voxel in the new treatment image belongs to the prostate or not. Among them, Gao et al. [8] trained a series of sparse representation classifiers and then used them to segment prostates with multi-atlas labeling. Li et al. [9] combined the auto-context model [10] and AdaBoost method to train the detectors for the prostate segmentation. Owing to the fact that these voxel-wise classification methods resorted to the prostate appearance characteristics of the patient-specific treatment images, they achieved satisfying performance in CT prostate segmentation.

Although the above methods have good performance in prostate segmentation, they always neglected the abundant prostate appearance characteristics and shape information of the current patient, which is embedded in the previous treatment images of the same patient. Therefore, if the valuable patient-specific information contained in the previous treatment images can be fully exploited, it can be expected that accuracy of prostate localization will increase significantly.

Motivated by the above ideas, we propose a CT prostate segmentation method which uses a continuously updated random forest model as the classifier. Specifically, after registering the training images of the same patient rigidly, we use the auto-context method to train a random forest model [11] as the classifier which will be utilized to learn the nonlinear mapping from local features of a voxel in the training images to its label; then, the trained classifier will be employed to classify each voxel in the new treatment image into the prostate or not. Once the prostate shape of the new treatment image has been segmented, the newly segmented prostate shape will be reviewed or adjusted by a clinician and successively added to the pool of the training images as the new patient-specific data. In this way, when the random forest model is retrained for the next treatment day, it can be updated with new prostate appearance characteristics and prostate shape information of the current patient. With more and more treatment images of the current patient acquired, this update scheme for the classifier can be continued during the therapy. The entire procedure of the proposed method is shown in Figure 2.

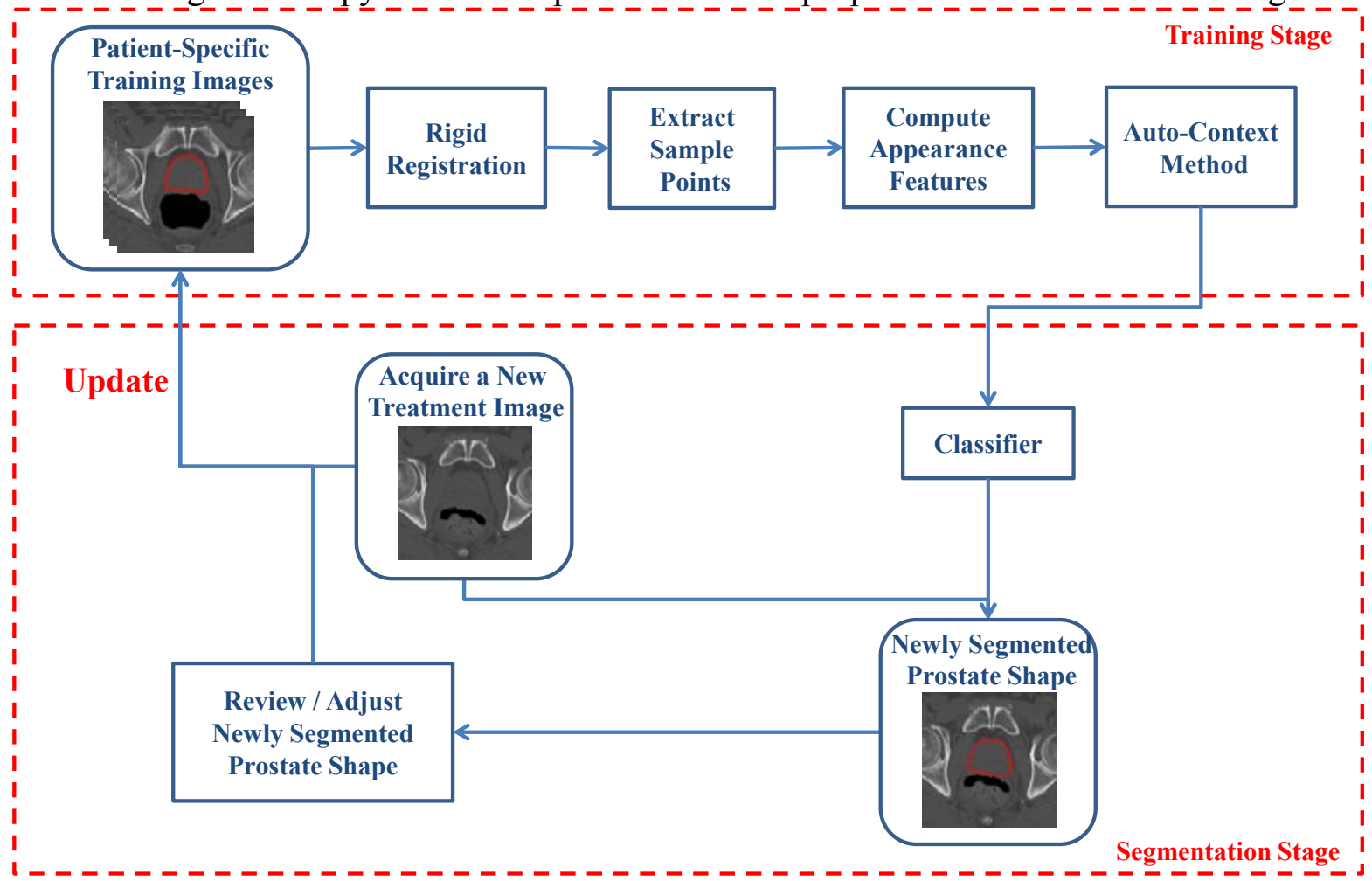

Fig. 2. The flowchart of the proposed method. 
In this manner, the abundant patient-specific information embedded in the previous treatment images can be fully used to reduce the variation of prostate shapes among different treatment days. And this continuously updated random forest model can better adapt to the current treatment image than ever before.

\section{Rigid Registration}

To eliminate the effect of the motion caused by the patient, we first need to register for the training images. Since the prostate motion or deformation of the same patient is not large through different treatment days, we assume that the rigid transformation is enough to describe the deformation between prostate shapes from different treatment days. Here, FLIRT method [12], which is widely used in rigid registration, is employed to implement rigid alignment.

\section{The Training of the Classifier}

After training images have been rigidly aligned, we use the auto-context method [10] to train a random forest model to identify which voxels belong to the prostate. The detailed steps of the training procedure by the auto-context method are given as follows:

(1) Compute a set of random Haar-like features to represent each sample point extracted from the training images. The definition of random Haar-like features is given as follows [2]:

$$
H\left(P_{I}\right)=\sum_{h=1}^{Z} p_{h} \sum_{\left\|\mathbf{X}-a_{h}\right\|_{\infty} \leq s_{h}} P_{I}(\mathbf{X})
$$

where $P_{I}$ is a local patch in image $I ; Z$ is the number of $3 \mathrm{D}$ cubic functions used in the Haar-like feature; $p_{h} \in\{-1,1\}, a_{h} \in \mathrm{R}^{3}$ and $s_{h}$ are the polarity, position and scale of the $h$-th $3 \mathrm{D}$ cubic function, respectively. By randomly sampling the number of $3 \mathrm{D}$ cubic functions, and the parameters of each cubic function in the Haar-like features, a set of random Haar-like features are generated, which will be treated as appearance features in the training stage;

(2) With the appearance features and the label values of each sample point in the training images, a classifier based on random forest model [11] is trained to learn the nonlinear mapping from local features of a voxel in the training images to its label. This classifier is named as the initial classifier.

(3) Exert the trained classifier to every training image for producing a classification probability map. Each value of the classification probability map points out the probability of being in the prostate for the corresponding voxel;

(4) Extract context features of the sample points from the classification probability map. The context features used in this paper can be referred to [9];

(5) Combine the extracted context feature with the appearance feature to train a new classifier;

(6) Exert the new classifier to every training image again to yield a new classification probability map for each training image;

(7) Repeat step (4)--(6) iteratively until each classification probability map will not improve significantly anymore;

(8) Save all the classifiers acquired in the different iterations as the final output of the training procedure.

\section{Segmentation Stage}

As the classifiers for the current patient has been achieved, it will be used to segment the prostate in the new treatment image which is acquired in the new treatment day. Before the segmentation, the FLIRT method should also be applied to the new treatment image for rigid alignment. The segmentation steps are described in details as follows:

(1) Use the random Haar-like features in Eq.(1) to calculate the appearance features of each voxel in the new treatment images; 
(2) Exert the initial classifier to the new treatment image with the appearance features and yield a classification probability map for it;

(3) Compute the context features for each voxel in the classification probability map acquired from the last step;

(4) Fuse the appearance features and the context features together as the input and exert the corresponding classifier to the new treatment image for computing a new classification probability map;

(5) Repeat step (3) --(4) iteratively until all the trained classifiers have been used.

(6) Apply a 3D level-set-based segmentation method [13] to the final classification probability map, and then obtain the final segmentation result of the new treatment image.

\section{The Update of the Classifiers}

Since the prostate shape in the current treatment image has been segmented, a clinician can review or even adjust the new prostate shape after finishing the radiotherapy at each treatment day. After that, we will add the reviewed or adjusted prostate shape into the patient-specific training pool to retrain the random forest model. With this retraining step, the random forest model can be updated.

While the next treatment day begins, the newly-updated random forest model will be used to segment the prostate shape in the new treatment images. It is worthy to be noted that the above prostate review/adjustment and the update of the random forest model will be repeated in the subsequent treatment days until the radiotherapy for the current patient is finished.

\section{Experimental Results}

In this section, the experiments are carried out to evaluate the performance of our proposed methods in terms of prostate segmentation.

Our experimental data consists of 91 images from 10 different patients which were scanned from a Siemens Somatom CT scanner. The resolution and image size of the acquired images are $0.98 \times 0.98 \times 3 \mathrm{~mm}^{3}$ and $512 \times 512 \times 30 \sim 120$ respectively. The prostates of all the images have been manually segmented as ground-truth by an experienced clinician in advance.

The same parameters of the random Haar-like features are applied in all the following experiments. We limit $Z$ to $\{1,2\}$ and $s_{h}$ to $\{3,5\}$; the patch size is set to $30 \times 30 \times 30$ voxel; $a_{h}$ can have arbitrary values as long as the $3 \mathrm{D}$ cubic function stays inside the local patch.

To evaluate accuracy of the prostate segmentation, we adopt the Dice ratio (Dice) [9] as the measurement.

Figure 3 shows some segmentation results (depicted in red contour) for three different patients, as well as their corresponding ground truth (depicted in blue contour). From Figure 3, we can see that in each patient, the prostate segmented by our method can match the ground truth successfully.

The mean Dice values for each patient are also listed in Table 1. Table 1 shows that our method can achieve satisfying Dice values for all the patients.

To validate that using continuously updated random forest model as the classifier contributes to the increase in accuracy of the prostate segmentation, the comparison of the mean Dice values between the segmented results obtained from the conventional random forest model (without updating) and the ones obtained from the continuously updated random forest model for each patient is provided in Figure 4. It can be observed from Figure 4 that for each patient, the mean Dice values from the continuously updated random forest model are pretty higher than the ones from the conventional random forest model. The advantage is especially obvious in the case of patient No. 7, 8 and 9. Thus, we conclude that the performance of the prostate segmentation can be improved by using continuously updated random forest model. 


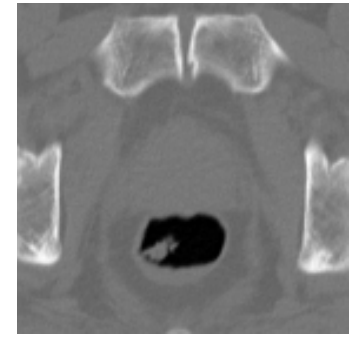

(a)

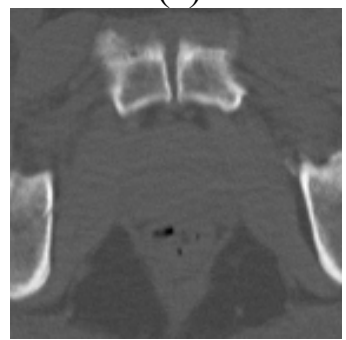

(d)

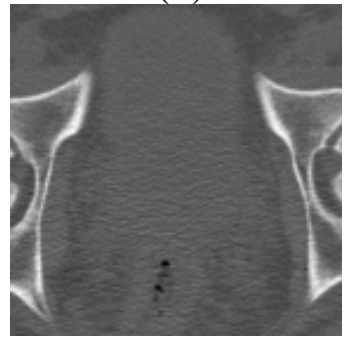

(g)

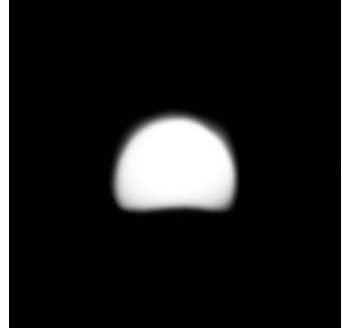

(b)

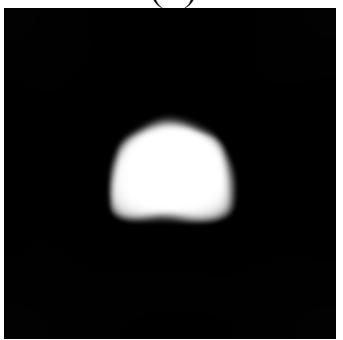

(e)

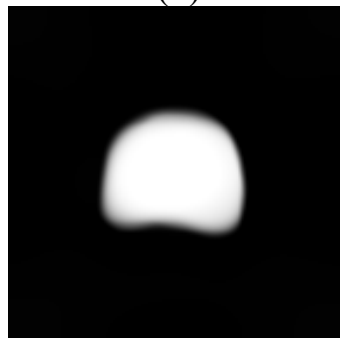

(h)

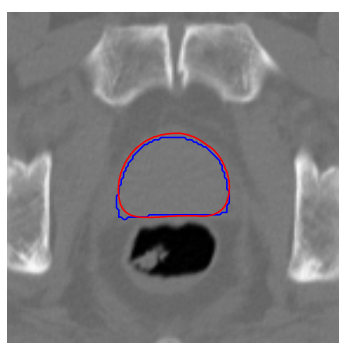

(c)

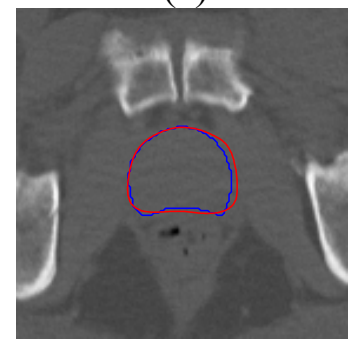

(f)

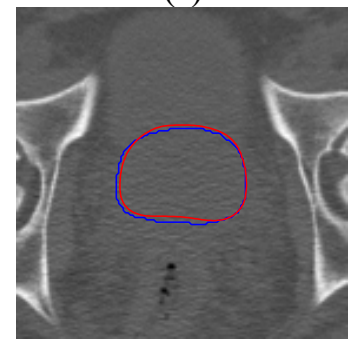

(i)

Fig.3. Some segmented results using our method for three different patients. The first column are the treatment images for patient A, B and C; the second column are the segmented results for patient A, B and $\mathrm{C}$; the third column are the the treatment images superimposed by the boundaries of the segmented results (depicted in red contour) and the ground truth (depicted in blue contour) for patient $\mathrm{A}, \mathrm{B}$ and $\mathrm{C}$.

Table 1. The mean Dice values of the segmented results using our method for 10 patients.

\begin{tabular}{|c|c|c|c|c|c|c|c|c|c|c|}
\hline Patient No. & 1 & 2 & 3 & 4 & 5 & 6 & 7 & 8 & 9 & 10 \\
\hline $\begin{array}{c}\text { Mean Dice } \\
\text { Values }\end{array}$ & 0.88 & 0.88 & 0.87 & 0.90 & 0.88 & 0.89 & 0.87 & 0.92 & 0.87 & 0.87 \\
\hline
\end{tabular}

- Conventional Update

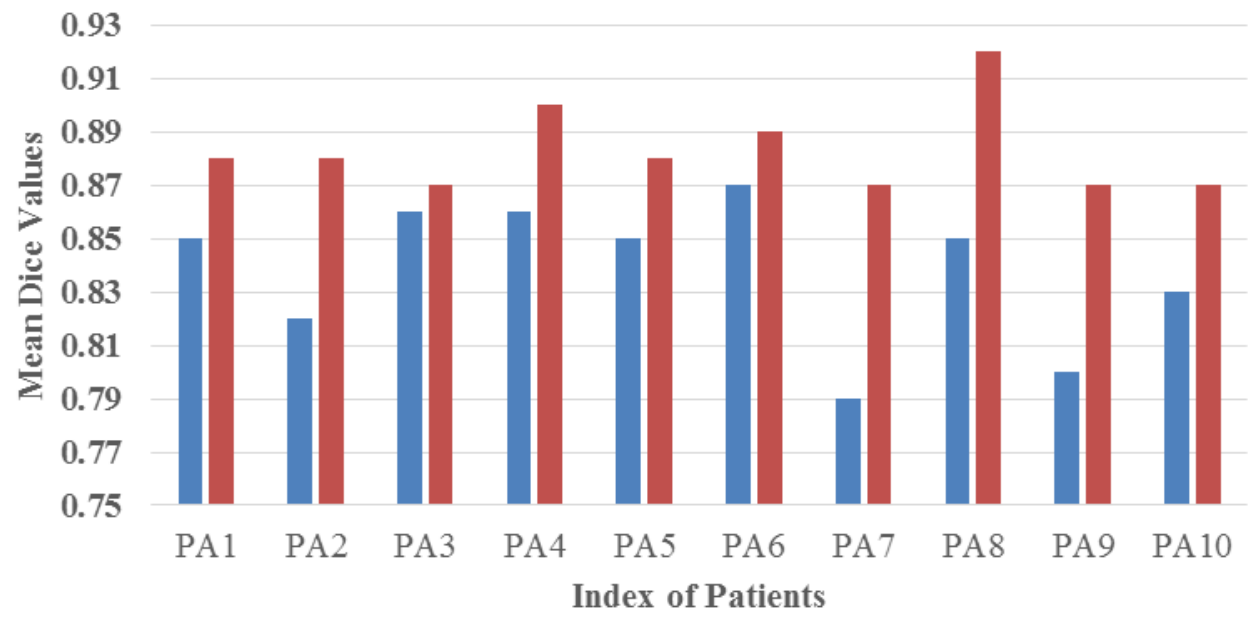

Fig.4. The comparison of the mean Dice values between the segmented results obtained from the conventional random forest model (without updating) and the ones obtained from the continuously updated random forest model for each patient. 


\section{Summary}

In this paper, we propose a novel prostate segmentation method for daily treatment CT images. It fully makes use of the prostate appearance and shape information embedded in the previous treatment images to train and update the random forest model. Furthermore, this update scheme can continue until the therapy for the patient completes. The experiments demonstrate that our proposed method can obtain satisfying segmented results for the treatment CT images.

\section{Acknowledgement}

This work was supported by the National Natural Science Foundation of China (Grants No. 31200747) and the Natural Science Foundation of Jiangsu Province (Grants No. BK2012437).

\section{References}

[1] Lei Xing. Overview of image-guided radiation therapy [J]. Official Journal of the American Association of Medical Dosimetrists, 200631 (1) 91-112.

[2] Yaozong Gao, Yang Zhan, Dinggang Shen. Incremental learning with selective memory (ILSM): towards fast prostate localization for image guided radiotherapy [J]. IEEE Transaction on Medical Imaging, 201433 (2) 518-534.

[3] María Jimena Costa, Hervé Delingette, Sébastien Novellas, Nicholas Ayache. Automatic Segmentation of Bladder and Prostate Using Coupled 3D Deformable Models [C]. Medical Image Computing and Computer-Assisted Intervention - MICCAI 2007, Springer, 2007.

[4] Daniel Freedman, Richard Radke, Tao Zhang, Yongwon Jeong, Michael Lovelock, George Chen. Model-based segmentation of medical imagery by matching distributions [J]. IEEE Transaction on Medical Imaging, 200524 (3) 281-292.

[5] Qianjing Feng, Mark Foskey, Dinggang Shen. Segmenting CT prostate images using population and patient-specific statistics for radiotherapy [J]. Medical Physics, 201037 (2) 4121-4132.

[6] Shu Liao, Dinggang Shen. A Feature-Based Learning Framework for Accurate Prostate Localization in CT Images [J]. IEEE Transaction on Image Processing, 201221 (8) 3546-3559.

[7] Mark Foskey, Brad Davis, Lucas Royal. Large Deformation Three-Dimensional Image Registration in Image-Guided Radiation Therapy [J]. Physics Medicine Biology, 200550 (24) 5869-5892.

[8] Yaozong Gao, Shu Liao, Dinggang Shen. Prostate segmentation on sparse representation based classification [J]. Medical Physics, 201239 (10) 6372-6387.

[9] Wei Li, Shu Liao, Qianjing Feng, Dinggang Shen. Learning image context for segmentation of the prostate in CT-guided radiotherapy [J]. Physics Medicine Biology, 201257 (5) 1283.

[10] Zuowen Tu, Xiang Bai. Auto-Context and Its Application to High-Level Vision Tasks and 3D Brain Image Segmentation [J]. IEEE Transaction on Pattern Analysis and Machine Intelligence, 2010 32 (10) 1744-1757.

[11] Leo Breiman. Random Forests [J]. Machine Learning, 200145 (1) 5-32.

[12] Bernd Fischer, Jan Modersitzki. FLIRT: A Flexible Image Registration Toolbox [J]. Biomedical Image Registration, 20032717 (1) 261-270.

[13] Tony Chan, Luminita Vese. Active contours without edges [J] IEEE Transaction on Image Processing, 200110 (2) 266-277. 